\title{
Tear film proteome in age-related macular degeneration
}

\author{
Mateusz Winiarczyk ${ }^{1} \cdot$ Kai Kaarniranta ${ }^{2} \cdot$ Stanisław Winiarczyk ${ }^{3} \cdot$ Łukasz Adaszek $^{3} \cdot$ Dagmara Winiarczyk $^{3}$. \\ Jerzy Mackiewicz ${ }^{1}$
}

Received: 1 September 2017 / Revised: 18 March 2018 / Accepted: 9 April 2018 /Published online: 25 April 2018

(C) The Author(s) 2018

\begin{abstract}
Purpose Age-related macular degeneration (AMD) is the main reason for blindness in elderly people in the developed countries. Current screening protocols have limitations in detecting the early signs of retinal degeneration. Therefore, it would be desirable to find novel biomarkers for early detection of AMD. Development of novel biomarkers would help in the prevention, diagnostics, and treatment of AMD. Proteomic analysis of tear film has shown promise in this research area. If an optimal set of biomarkers could be obtained from accessible body fluids, it would represent a reliable way to monitor disease progression and response to novel therapies.

Methods Tear films were collected on Schirmer strips from a total of 22 patients ( 8 with wet AMD, 6 with dry AMD, and 8 control individuals). 2D electrophoresis was used to separate tear film proteins prior to their identification with matrix-assisted laser desorption/ionization time of flight spectrometer (MALDI-TOF/TOF) and matching with functional databases.

Results A total of 342 proteins were identified. Most of them were previously described in various proteomic studies concerning AMD. Shootin-1, histatin-3, fidgetin-like protein 1, SRC kinase signaling inhibitor, Graves disease carrier protein, actin cytoplasmic 1, prolactin-inducible protein 1, and protein S100-A7A were upregulated in the tear film samples isolated from AMD patients and were not previously linked with this disease in any proteomic analysis.

Conclusion The upregulated proteins supplement our current knowledge of AMD pathogenesis, providing evidence that certain specific proteins are expressed into the tear film in AMD. As far we are aware, this is the first study to have undertaken a comprehensive in-depth analysis of the human tear film proteome in AMD patients.
\end{abstract}

Keywords Age-related macular degeneration $\cdot$ Biomarker $\cdot$ Proteomics $\cdot$ Tear film

\section{Introduction}

According to WHO, 285 million people are estimated to be visually impaired worldwide: 39 million are blind and 246 million have poor vision (www.who.int/mediacentre/

Electronic supplementary material The online version of this article (https://doi.org/10.1007/s00417-018-3984-y) contains supplementary material, which is available to authorized users.

Jerzy Mackiewicz

jerzymackiewicz@umlub.pl

1 Department of Vitreoretinal Surgery, Medical University of Lublin, Lublin, Poland

2 Department of Ophthalmology, University of Eastern Finland and Kuopio University Hospital, Kuopio, Finland

3 Department of Epizootiology, University of Life Sciences of Lublin, Lublin, Poland factsheets/fs282/). AMD is the leading cause of blindness in the elderly population in the Western countries. The latest recent study revealed that early AMD signs can be detected already in patients younger than 30 years of age [1]. Due to the absence of an effective preventive treatment, the number of patients severely disabled by AMD is expected to increase by more than $50 \%$ in the next 20 years. The disease not only exerts a tremendous impact on the physical and mental health of the geriatric population and their families but it is also becoming a major public health and financial burden [2].

AMD is characterized by a progressive loss of central vision; this is attributable to degenerative and neovascular changes in the macula, the highly specialized region of the central retina responsible for fine and color vision [3]. AMD is divided into the slowly developing dry ( $80-85 \%$ of cases) and the rapidly blinding wet (15-20\%) forms. The prevalence of AMD increases with age; in the age-category of 65-74, its prevalence is $15 \%$; from 75 to 84 , it grows to $25 \%$; and in individuals aged 85 and older, the prevalence rises to 
$30 \%$. A reasonable overall estimate of the incidence of advanced AMD in persons aged 65-74 years is $1 \%$, increasing to $5 \%$ in the age-category of 75-84 years, and further up to $13 \%$ in subjects 85 years old and above [4]. In Poland, 14,000 new AMD patients are diagnosed each year. AMD has been commonly called "the blindness epidemic," (http://retinaamd. org.pl/wp-content/uploads/2016/02/Audytraport.pdf).

The etiology of AMD is known to be multifactorial [5]. In addition to a strong genetic component (i.e., defects in certain genes, e.g., ARMS, CFH, C3), and aging, there are thought to be environmental risk factors such as smoking, obesity, hypertension, and hypercholesterolemia which predispose to AMD [4] The macula area of the retina is a region with a high metabolic rate; it is also subjected to a high oxygen pressure and redox reactions continuously generating highly reactive radicals. It is widely believed that the presence of chronic oxidative stress, impaired autophagy, and inflammation are all strongly linked to the pathogenesis of AMD [3]. The combination of those processes ultimately leads to the death of the RPE cells $[6,7]$. One hallmark of AMD is the detrimental accumulation of lysosomal lipofuscin in the RPE cells and the build-up of extracellular drusen deposits between the basal lamina of the RPE and the inner collagenous layer of Bruch's membrane. High amounts of lipofuscin and drusen deposits predict AMD progression and severity. Choroidal neovascularization, a hallmark of wet AMD, may develop in certain AMD cases. If not treated, these pathological processes promote fibrogliosis, resulting in the production of a disciform scar and severe loss of vision. The available treatment options, such as anti-VEGF intravitreal injections, are useful only in patients suffering from wet AMD and can only delay the progression of the disease [3]. One major public health challenge is to devise an effective primary prevention strategy for identifying highrisk AMD patients and to improve current follow-up as well as discovering more effective treatments. However, in order to achieve these goals, a better understanding of AMD's pathophysiological details is required.

The primary clinical hallmark of AMD is RPE degeneration. The post-mitotic RPE cells in the macula are constantly exposed to a high metabolic and oxidative stress due to the daily heterophagic processing of the high concentration of polyunsaturated fatty acids (PUFAs) originating from the retinal outer segments, the vigorous choriocapillaris circulation, and the constant exposure to light [3]. During the aging of RPE cells, their capacity to neutralize mitochondrial-derived reactive oxidative species (ROS) diminishes due to a decline in antioxidant production, impaired DNA damage or protein repair processes, and disturbed proteolysis $[8,9]$. The intense ROS production and impaired defense systems further damage cellular proteins accentuating the detrimental protein aggregation. The production of lysosomal lipofuscin is one example of the disturbed proteostasis in RPE cells [10-12].
Oxidized retinal outer segment discs are not efficiently digested in the lysosomes of aged RPE cells, instead, they become deposited in the form of lipofuscin. The autofluorescent lipofuscin is a complex heterogeneous mixture of lipid-protein aggregates, which sensitizes RPE cells to light and increases oxidative stress, ultimately evoking misfolding of proteins. The heat-shock protein (Hsp) stress response is the only system capable of refolding misfolded proteins, a crucial mechanism promoting cellular survival in times of oxidative stress [13]. The upregulation of Hsps has been detected in RPE homogenates isolated from human donor AMD samples $[14,15]$. This is not only an indication of the over-stressed RPE cells, but it also highlights the dysfunction of the proteasomal clearance [16]. Once Hsps' repair capacity has become exceeded, single peptide species can be degraded by the proteasome, while larger aggregates are broken down by selective macroautophagy. Starvation, hypoxia, oxidative stress, and protein aggregation are well-known factors for inducing autophagy $[3,17,18]$. Autophagy is a fundamental mechanism for reducing the amount and toxicity of the protein aggregates and to maintain retinoid levels to support vision $[17,18]$. However, the autophagy activity is known to be reduced during the aging process of the RPE cells and in AMD $[9,17,18]$.

A tear film covers the surface of the eye, providing protection, lubrication, and nutrition. It consists of three layers: the outermost lipid layer, the intermediate aqueous layer, and the innermost mucus layer. The tear film is produced by lacrimal glands and Meibomian glands, accessory glands, and goblet cells [19]. Since it is readily accessible, it has been a target in proteomic programs investigating various ocular and systemic diseases such as dry eye syndrome [20-22], diabetes [23, 24], Parkinson's disease, multiple sclerosis, or cancer [25].

Previous ocular proteome studies in AMD have been focused on retina, vitreous body, aqueous humor, or blood, with multiple proteins identified in each of them [26-42]. Post-mortem retinal samples show differences between protein distribution between central and peripheral retina in AMD [27], also drusen proteomic composition seems to be different in healthy individuals and AMD patients [40]. Yuan et al. showed elevated levels of metalloproteinase inhibitor 3, vitronectin, C9, clusterin, $\alpha$-crystallin B, protein-glutamine $\gamma$-glutamyltransferase 2 , and $\mathrm{C} 3$ in the Bruchs membrane-choroid complex obtained from human donors [43].

Ecker et al. identified multiple inflammation-related proteins in the vitreous samples [27]. Matrix metalloproteinase9 (MMP9) was elevated together with increased subretinal fluid [44]. In other studies by Koss et al. and Nobl et al., where vitreous samples were also obtained, alpha-1antitrypsin, fibrinogen alpha chain, prostaglandin H2 Disomerase, opticin, pigment epithelium-derived factor, and 
prostaglandin-H2 D-isomerase were also linked with AMD pathogenesis $[28,29]$.

In aqueous humor (AH) analyzed by MRM-MS techniques, plasma protease $\mathrm{C} 1$ inhibitor, TGFB1, ceruloplas$\mathrm{min}$, and pigment-epithelium-derived factor were reported to be significantly different between control and AMD group [31]. Yao et al. reported that the AH protein composition was significantly different between wet AMD and non-AMD patients, with 78 differentiating proteins (68 identified). They proposed crystallins, lipocalin-1, and cyclooxygenase- 2 as key players in AMD pathogenesis [30]. Kang et al. selected six proteins as potential biomarkers in AH: actin, myosin-9, Hsp-70, cathepsin D, and cytokeratin 8 and 14 [36]. In more recent study, $\mathrm{Ng}$ et al. showed correlation between high angiopoietin-2 levels with disease severity at presentation. Hepatocyte growth factor (HGF), interleukin 8, and TIMP-1 levels were also elevated in AMD group [37]. Interestingly, those findings are in contrary to previous reports of lower angiopoietin 2 levels in AMD patientst [45].

In blood samples obtained from AMD patients, noticeable differences between analyzed groups were also observed. Iannaccone et al. suggested that circulating autoantibodies play a role in AMD pathogenesis, and five of the possible autoreactivity targets were conclusively identified: two members of the heat-shock protein 70 (HSP70) family, HSPA8 and HSPA9; another member of the HSP family, HSPB4, also known as alpha-crystallin A chain (CRYAA); Annexin A5 (ANXA5); and Protein S100-A9, also known as calgranulin B [32, 33]. Kim and et al. in proposed vinculin, phospholipid transfer protein (PLTP), and mannan-binding lectine serine protease 1 (MASP-1) as potential AMD biomarkers, with high AUC values (i.e., 0.936 for PLTP) [38, 41]. Xu et al. identified 28 proteins that were elevated in AMD patients compared to control group [42].

While most of the abovementioned studies propose different AMD biomarkers, proteins identified as differentiating are usually involved in inflammation, impaired autophagy, neovascularization, and oxidative stress response. All of those are well documented as key processes in AMD development. Invasively taken samples are hard to reproduce in the same patients group. This feature reduces their usefulness for patient monitoring and validating the obtained results. Tear film can be taken from the same patient numerous times, even by each appointment.

Our plan was to perform first comprehensive analysis of tear film proteome in AMD patients. To our best knowledge, there is presently no study that would evaluate deeply tears protein composition in AMD. All the other AMD proteomic studies present similar set of expressed proteins, involved in inflammation, microtubule organization, and protein refolding processes. We hypothesize similar set of proteins might be present in tear film.

\section{Materials and methods}

The study was approved by Bioethical Committee of Medical University of Lublin by declaration number KE-0254/238/ 2015. Informed consent was obtained from every patient enrolled in the study. The purpose and design of the study, as well as its possible complications, were explained to every patient, and written consents were obtained. All experiments followed the provisions of the Declaration of Helsinki.

Patients with wet or dry AMD as well as a control group of cataract patients were included in this single-center prospective study. We applied the following exclusion criteria: eyelid problems (entropion, ectropion, Meibomian Gland Dysfunction and other anterior segment disorders), use of contact lenses, amaurosis, patients suffering from eye trauma, corneal ulcer, uveitis, ophthalmic surgery excluding phacoemulsification, diabetes mellitus. Hypertension was not an exclusion criterion if it did not result in signs of hypertensive retinopathy.

There were 22 patients included in the study; 8 patients with wet AMD, 6 with dry AMD, and 8 were in the control group. Sex distribution was similar between men and women in each group. All criteria-satisfying patients underwent a full ophthalmic examination by the same ophthalmologist consisting of visual acuity test, slit lamp examination, intraocular pressure measurement, optical coherent tomography, and fluorescent angiography if needed.

As ocular surface disease would disturb the results, each patient's eyelids and anterior segment were carefully assessed. Meibomian Gland Dysfunction was absent in all patients, tear film break-up time (BUT) was within normal limits (over $10 \mathrm{~s}$ ), and all the patients had Schirmer test result over $13 \mathrm{~mm}$ in $5 \mathrm{~min}$.

The wet AMD consisted of eight patients, four men and four women with mean age of $78.1(\mathrm{SD}=6.73)$. Mean best corrected visual acuity (BCVA) was 0.25 for right eye ( $\mathrm{SD}=$ $0.21)$ and 0.18 for left eye $(S D=0.1)$ in Snellen charts. Mean intraocular pressure (IOP) was 14.1 for right eye $(\mathrm{SD}=3.04)$ and 14.38 for left eye $(\mathrm{SD}=3.29)$. Schirmer test result in 5 min time was $17.5 \mathrm{~mm}(\mathrm{SD}=2.39)$ for right and $17.36 \mathrm{~mm}(\mathrm{SD}=2.39)$ for left eye. On slit lamp examination, patients presented active form of AMD in at least one eye with intra/subretinal fluid presence. Seven of them were previously treated with anti-VEGF therapy in one or both eyes, and one patient has undergone a cataract surgery. One patient presented naïve form of wet AMD, with no previous treatment. All the clinical findings were validated by fundus photographs and fluorescein angiography combined with SD-OCT. Environmental risk factors, BMI, smoking, and systemic diseases, were assessed. Average BMI was $26.99(\mathrm{SD}=2.65)$ in wet AMD cases. Five patients were active or previous smokers; six were treated for hypertension and two for cardiovascular disease. All the patients are shown in Table 1. 
Table 1 Wet AMD patients

\begin{tabular}{|c|c|c|c|c|c|c|c|c|}
\hline $\begin{array}{l}\text { Sex/ } \\
\text { age }\end{array}$ & $\begin{array}{l}\text { BCVA OD/OS } \\
\text { (Snellen) }\end{array}$ & $\begin{array}{l}\text { IOP }(\mathrm{mmHg}) \\
\text { OD/OS }\end{array}$ & $\begin{array}{l}\text { Schirmer test result (mm } \\
\text { in } 5 \mathrm{~min} \text { ) }\end{array}$ & $\mathrm{SRF} / \mathrm{CRT}(\mu \mathrm{m}) / \mathrm{PED}$ & $\begin{array}{l}\text { Ocular } \\
\text { interventions }\end{array}$ & BMI & $\begin{array}{l}\text { Smoking } \\
\text { (pack-year) }\end{array}$ & $\begin{array}{l}\text { Systemic } \\
\text { diseases }\end{array}$ \\
\hline M76 & $0.1 / 0.1$ & $14 / 16$ & $20 / 20$ & $\begin{array}{l}\text { OS_SRF(+), CRT 326, } \\
\text { PED }(+) \\
\text { OD_macular scar }\end{array}$ & - & 28.1 & 30 & HT, CVD \\
\hline F76 & $0.2 / \mathrm{CF} 0.5 \mathrm{~m}$ & $12 / 12$ & $18 / 20$ & $\begin{array}{l}\text { OD_drusen }(+++) \\
\text { OS-SRF }(+), \text { CRT } 330\end{array}$ & $\begin{array}{l}\text { Anty-VEGF } 4 \times \\
\text { do OS }\end{array}$ & 30.1 & - & HT, CVD \\
\hline F77 & $0.5 / 0.2$ & $15 / 12$ & $20 / 20$ & $\begin{array}{l}\text { OD-normal } \\
\text { OS-SRF(+), CRT } 331\end{array}$ & $\begin{array}{l}\text { Anty-VEGF } 2 \times \\
\text { do OS }\end{array}$ & 27.3 & - & HT \\
\hline M91 & $0.1 / 0.2$ & $19 / 20$ & $14 / 16$ & $\begin{array}{l}\text { OD_SRF(+), CRT } 248 \\
\text { OS-macular scar }\end{array}$ & $\begin{array}{l}\text { Anty-VEGF } \\
\text { ODS } 5 \times\end{array}$ & 22.2 & 40 & - \\
\hline F77 & $0.5 / 0.3$ & $9 / 11$ & $18 / 15$ & $\begin{array}{l}\text { OD_normal } \\
\text { OS-SRF(+), CRT } 421\end{array}$ & $\begin{array}{l}\text { Anty-VEGF } \\
\text { OS } 1 \times\end{array}$ & 29.5 & - & HT \\
\hline M73 & $0.1 / 0.2$ & $13 / 12$ & $14 / 14$ & $\begin{array}{l}\text { OD_SRF(+), CRT } 230 \\
\text { OS-macular scar }\end{array}$ & $\begin{array}{l}\text { Phaco + ILCP } \\
\text { ODS } \\
\text { Anty-VEGF } \\
\text { ODS } 3 \times\end{array}$ & 25.4 & 20 & - \\
\hline M70 & CF $1 \mathrm{~m} / 0.1$ & $17 / 18$ & $19 / 17$ & $\begin{array}{l}\text { OD_-subretinal } \\
\text { hemorrhage + CNV } \\
\text { OS-macular scar }\end{array}$ & $\begin{array}{l}\text { Anty-VEGF } \\
\text { ODS } 4 \times\end{array}$ & 24.9 & 30 & HT \\
\hline F85 & $0.5 / 0.3$ & $14 / 14$ & $17 / 17$ & $\begin{array}{l}\text { OD-drusen(+++) } \\
\text { OS-SRF(+), CRT } 389\end{array}$ & $\begin{array}{l}\text { Anty-VEGF } \\
\text { OS } 3 \times\end{array}$ & 28.4 & 10 & HT \\
\hline
\end{tabular}

The dry AMD group consisted of six patients, three men and three women with mean age of 76.3 ( $\mathrm{SD}=5.0)$. Mean best corrected visual acuity (BCVA) was 0.18 for right eye $(\mathrm{SD}=0.17)$ and 0.27 for left eye $(\mathrm{SD}=0.12)$ in Snellen charts. Mean intraocular pressure (IOP) was 12.7 for right eye $(\mathrm{SD}=$ 2.6) and 12.2 for left eye $(\mathrm{SD}=1.7)$. Schirmer test result in 5min time was $17.0 \mathrm{~mm}(\mathrm{SD}=1.79)$ for right and $18.33 \mathrm{~mm}$ $(\mathrm{SD}=1.63)$ for left eye. On slit lamp examination, they presented either confluent drusen or geographic atrophy. None of the patients has undergone any ocular surgical intervention. All the clinical findings were validated by fundus photographs and SD-OCT. In the dry AMD group, average BMI was 25.6 $(\mathrm{SD}=3.15)$. Two patients were active or previous smokers; four were treated for hypertension and two for cardiovascular disease. All the patients are shown in Table 2.

Control group consisted of eight patients, three men and five women with mean age of $75(\mathrm{SD}=3.0)$. Mean best corrected visual acuity (BCVA) was 0.6 for right eye $(\mathrm{SD}=$
$0.3)$ and 0.59 for left eye $(\mathrm{SD}=0.26)$ in Snellen charts. Mean intraocular pressure (IOP) was 13.13 for right eye $(\mathrm{SD}=3.23$ ) and 12.86 for left eye $(\mathrm{SD}=1.73)$. Schirmer test result in 5min time was $16.63 \mathrm{~mm}(\mathrm{SD}=2.20)$ for right and $17.0 \mathrm{~mm}$ $(\mathrm{SD}=2.20)$ for left eye. On slit lamp examination, there were no abnormalities in either the anterior or posterior segment. The control group was recruited from the patients qualified for standard cataract surgery. Three of those patients were pseudophakic, with surgery performed at least 1 year ago. Posterior pole was evaluated by fundus photograph and SDOCT. In the control group, average BMI was 27.4 ( $\mathrm{SD}=2.6$ ). Five patients were active or previous smokers; five were treated for hypertension and three for cardiovascular disease. All the patients are shown in Table 3.

Tear film was collected from each eye onto a Schirmer strip (TearFlo, HUB Pharmaceuticals LLC [46, 47]. Each collection was performed by the author, in the morning hours, between 8 and 11 a.m. If fluorescein angiography was

Table 2 Dry AMD patients

\begin{tabular}{|c|c|c|c|c|c|c|c|c|}
\hline Sex/age & $\begin{array}{l}\text { BCVA OD/OS } \\
\text { (Snellen) }\end{array}$ & $\begin{array}{l}\text { IOP }(\mathrm{mmHG}) \\
\text { OD/OS }\end{array}$ & $\begin{array}{l}\text { Schirmer test result } \\
\text { (mm in } 5 \mathrm{~min})\end{array}$ & $\begin{array}{l}\text { Macula } \\
\text { condition }\end{array}$ & $\begin{array}{l}\text { Ocular } \\
\text { interventions }\end{array}$ & BMI & $\begin{array}{l}\text { Smoking } \\
\text { (pack-year) }\end{array}$ & $\begin{array}{l}\text { Systemic } \\
\text { diseases }\end{array}$ \\
\hline F79 & $0.1 / 0.4$ & $11 / 10$ & $18 / 19$ & $\begin{array}{l}\text { OD - GA } \\
\text { OS - confluent drusen }\end{array}$ & - & 27.8 & - & - \\
\hline M72 & $0.2 / 0.4$ & $13 / 13$ & $20 / 20$ & ODS - confluent drusen & - & 22.9 & 40 & - \\
\hline F69 & $0.4 / 0.3$ & $11 / 11$ & $16 / 20$ & ODS — confluent drusen & - & 24.1 & - & HT \\
\hline F80 & $0.1 / 0.2$ & $17 / 14$ & $16 / 16$ & $\begin{array}{l}\mathrm{OD}-\mathrm{GA} \\
\mathrm{OS} \text { - confluent drusen }\end{array}$ & - & 21.8 & - & HT, CVD \\
\hline M82 & $0.2 / 0.2$ & $14 / 14$ & $15 / 17$ & ODS — confluent drusen & - & 29.8 & - & HT, CVD \\
\hline M76 & $0.1 / 0.1$ & $10 / 11$ & $17 / 18$ & ODS-GA & - & 27.4 & 30 & HT \\
\hline
\end{tabular}


Table 3 Control group patients

\begin{tabular}{|c|c|c|c|c|c|c|c|}
\hline $\begin{array}{l}\text { Sex/ } \\
\text { age }\end{array}$ & $\begin{array}{l}\text { Visus } \\
\text { BCVA OD/ } \\
\text { OS }\end{array}$ & $\begin{array}{l}\text { IOP } \\
(\mathrm{mmHG})\end{array}$ & $\begin{array}{l}\text { Schirmer test result } \\
(\mathrm{mm} \text { in } 5 \mathrm{~min})\end{array}$ & $\begin{array}{l}\text { Ocular } \\
\text { interventions }\end{array}$ & BMI & $\begin{array}{l}\text { Smoking } \\
\text { (pack-years) }\end{array}$ & $\begin{array}{l}\text { Systemic } \\
\text { diseases }\end{array}$ \\
\hline & M80 & $1.0 / 0.8$ & $12 / 12$ & $13 / 16$ & & $\begin{array}{l}\text { Phaco + } \\
\text { ILCP OD }\end{array}$ & 23.5 \\
\hline 35 & HT, CVD & & & & & & \\
\hline F74 & $0.6 / 0.7$ & $12 / 13$ & $20 / 19$ & - & 28.1 & - & HT,CVD \\
\hline \multirow[t]{2}{*}{ F73 } & $0.9 / 0.6$ & $16 / 13$ & $17 / 18$ & - & 30.4 & - & HT \\
\hline & M77 & $0.9 / 0.5$ & $10 / 12$ & $15 / 14$ & & $\begin{array}{l}\text { Phaco + } \\
\text { ILCP OD }\end{array}$ & 29.1 \\
\hline 80 & HT & & & & & & \\
\hline F74 & $0.5 / 0.6$ & $15 / 14$ & $18 / 20$ & - & 28.9 & 20 & HT \\
\hline F73 & $0.2 / 0.2$ & $19 / 16$ & $17 / 14$ & - & 29.4 & 10 & - \\
\hline F71 & $0.3 / 1.0$ & $10 / 10$ & $18 / 18$ & $\begin{array}{l}\text { Phaco + } \\
\text { ILCP OS }\end{array}$ & 24.2 & - & CVD \\
\hline & M78 & $0.4 / 0.3$ & $11 / 13$ & $15 / 17$ & - & 25.6 & 13 \\
\hline
\end{tabular}

performed, material was collected always beforehand. Sterile gloves were always used by the investigator. Schirmer strips were placed into the lower sacs of both eyes at $1 / 3$ of the distance of the eyelid from the nasal canthus without anesthesia. This method was chosen over a microcapillary, flush tear, or basal tear collection mainly due to the patient comfort. As a standard clinical procedure, it is usually well known and tolerated by the patients. There is currently no consensus about which method of collection should be used for proteomic analysis [48-51]. After holding the strips in place for $5 \mathrm{~min}$, they were removed, transferred to a $1.5-\mathrm{mL}$ Eppendorf tube without any buffer, and were then immediately frozen at $80^{\circ} \mathrm{C}$. In the next step, the proteins were eluted from the strips into $8 \mathrm{M}$ urea buffer containing 3\% CHAPS detergent and $25 \mathrm{mM}$ dithiothreitol (DTT), $30 \mu \mathrm{g}$ of protease inhibitor cocktail, after which the samples were desalted and concentrated through Amicon® Ultra $0.5-\mathrm{mL}$ centrifugal filters $3 \mathrm{kDa}$ (Merck). The protein concentration was determined in a Direct Detect $\circledast$ infrared spectrometer (Merck).

In the 2D electrophoresis, the sample buffer (Bio-Rad) was added to a sample containing of $40 \mu \mathrm{g}$ total protein. Next, the obtained solution was placed with the immobilized $\mathrm{pH}$ gradient (IPG) strip (17 cm, pH 3-10, linear pH gradient, Bio-Rad) in a rehydrating plate for isoelectric focusing with the Hoefer IEF100 instrument. The IPG strips were incubated for $15 \mathrm{~min}$ in equilibration buffer (50 mM Tris- $\mathrm{HCl}, \mathrm{pH} 8.8,6 \mathrm{M}$ urea, $30 \%$ glycerol, $2 \%$ SDS) containing 1\% DTT, after which the incubation was continued for $15 \mathrm{~min}$ in equilibration buffer containing $2.5 \%$ iodoacetamide. The second electrophoretic separation was conducted using $12.5 \%$ polyacrylamide gel in BIO-RAD PROTEAN II xi Cell. Vertical separation was achieved with $600 \mathrm{~V} / 50 \mathrm{~mA} / 30 \mathrm{~W}$ in $0.025 \mathrm{M}$ Tris/GLY pH 8.3 buffer. After electrophoretic separation, proteins were silver stained according to Shevchenko et al. [52]. Initial quantitative and qualitative analyses were performed by comparing the electrophoretic gels from all the groups.
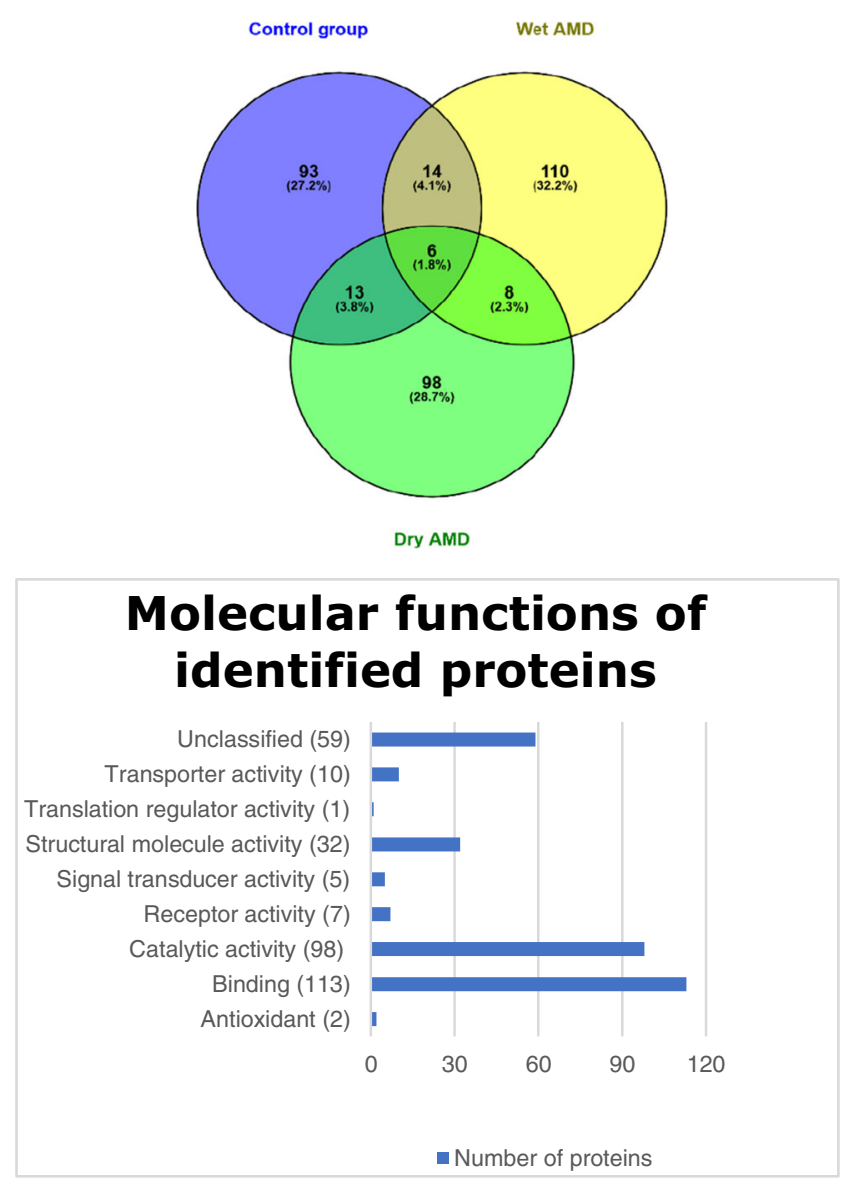

Fig. 1 The upper figure represents the number of identified proteins with their distribution and the overlap between the analyzed groups. The lower chart describes the molecular function of all identified proteins based on http://pantherdb.org gene ontology online tool 
Table 4 Proteins specific for wet AMD with related pathways

\section{Protein}

Pathway (by PANTHER classification)

Angiogenesis related

Ras GTPase-activating protein 1 (RASA1)

PDGF signaling pathway

Signal transducer and activator of transcription 3 (STAT3)

Fibroblast growth factor receptor 1 (FGFR1)

Ras GTPase-activating protein 1 (RASA1)

Signal transducer and activator of transcription 3 (STAT3)

Fibroblast growth factor receptor 1 (FGFR1)

Ras GTPase-activating protein 1 (RASA1)

Calcium/calmodulin-dependent 3',5'-cyclic nucleotide phosphodiesterase 1A (PDE1A)

Signal transducer and activator of transcription 3 (STAT3)

Ras GTPase-activating protein 1 (RASA1)

Signal transducer and activator of transcription 3 (STAT3)

Signal transducer and activator of transcription 3 (STAT3)

Apoptosis related

Insulin-like growth factor-binding protein 3 (IGFBP3)

Cyclin-G1 (CCNG1)

Cyclin-dependent kinase 4 inhibitor D (CDKN2D)

Cyclin-G1 (CCNG1)

Bcl-2-related ovarian killer protein (BOK)

Bcl-2-like protein 10 (BCL2L10)

Trafficking

Synaptotagmin-like protein 3 (SYTL3)

Inflammation

Myosin-13 (MYH13)

Signal transducer and activator of transcription 3 (STAT3)

Ras GTPase-activating protein 1 (RASA1)

Signal transducer and activator of transcription 3 (STAT3)

Signal transducer and activator of transcription 3 (STAT3)

Cytoskeleton and cell motility

Myosin-13 (MYH13)

Collagen alpha-1(IX) chain (COL9A1)

Other

Ras GTPase-activating protein 1 (RASA1)

Signal transducer and activator of transcription 3 (STAT3)

Myosin-13 (MYH13)

Rod cGMP-specific 3',5'-cyclic phosphodiesterase subunit beta (PDE6B)

Phosphorylase $\mathrm{b}$ kinase regulatory subunit beta (PHKB)

Retinal dehydrogenase 1 (ALDH1A1)

Myosin-13 (MYH13)
Angiogenesis

FGF signaling pathway

CCKR signaling map

EGF receptor signaling pathway

Gonadotropin-releasing hormone receptor pathway

p53 pathway

p53 pathway feedback loops 2

Apoptosis signaling pathway

Synaptic vesicle trafficking

Inflammation mediated by chemokine and cytokine signaling pathway

Interleukin signaling pathway

JAK/STAT signaling pathway

Cytoskeletal regulation by Rho GTPase Integrin signaling pathway

Insulin/IGF pathway-mitogen activated protein kinase kinase/MAP kinase cascade

Ras Pathway

Nicotinic acetylcholine receptor signaling pathway

Heterotrimeric G-protein signaling pathway-rod outer segment phototransduction

Heterotrimeric G-protein signaling pathway-Gi alpha and Gs alpha-mediated pathway

5-hydroxytryptamine degradation

Wnt signaling pathway 
Table 5 Proteins specific for dry AMD with related pathways
Protein

Pathway (by PANTHER classification)

Oxidative stress, inflammation, and proteolysis related

Guanine nucleotide-binding protein subunit alpha-11 (GNA11)

Myc proto-oncogene protein (MYC)

Ribosomal protein S6 kinase alpha-3 (RPS6KA3)

Myc proto-oncogene protein (MYC)

Dual specificity protein phosphatase 22 (DUSP22)

Guanine nucleotide-binding protein subunit alpha-11 (GNA11)

Guanine nucleotide-binding protein subunit alpha-11 (GNA11)

Guanine nucleotide-binding protein subunit alpha-11 (GNA11)

Myc proto-oncogene protein (MYC)

Ribosomal protein S6 kinase alpha-3 (RPS6KA3)

Guanine nucleotide-binding protein subunit alpha-11 (GNA11)

cGMP-dependent protein kinase 2 (PRKG2)

Endothelin-converting enzyme 1 (ECE1)

Guanine nucleotide-binding protein subunit alpha-11 (GNA11)

Fibroblast growth factor 9 (FGF9)

Pyruvate kinase PKM (PKM)

5'-AMP-activated protein kinase subunit gamma-2 (PRKAG2)

Guanine nucleotide-binding protein subunit alpha-11 (GNA11)

F-box/WD repeat-containing protein $1 \mathrm{~A}$ (BTRC)

Guanine nucleotide-binding protein subunit alpha-11 (GNA11)

Calpain-7 (CAPN7)

AP-2 complex subunit alpha-1 (AP2A1)

Cytoplasmic dynein 1 light intermediate chain 1 (DYNC1LI1)

Ribosomal protein S6 kinase alpha-3 (RPS6KA3)

Laminin subunit alpha-3 (LAMA3)

Glutamate receptor 2 (GRIA2)

Guanine nucleotide-binding protein subunit alpha-11 (GNA11)

Glutamate receptor 2 (GRIA2)

Guanine nucleotide-binding protein subunit alpha-11 (GNA11)

Guanine nucleotide-binding protein subunit alpha-11 (GNA11)

Myc proto-oncogene protein (MYC)

Ribosomal protein S6 kinase alpha-3 (RPS6KA3)

Guanine nucleotide-binding protein subunit alpha-11 (GNA11)

Ubiquitin/ISG15-conjugating enzyme E2 L6 (UBE2L6)

Pyruvate kinase PKM (PKM)

PI3 kinase pathway
Inflammation mediated by chemokine and cytokine signaling pathway

Interleukin signaling pathway

Oxidative stress response

Histamine $\mathrm{H} 1$ receptor-mediated signaling pathway

5HT2 type receptor-mediated signaling pathway

Alpha adrenergic receptor signaling pathway

CCKR signaling map

Corticotropin releasing factor receptor signaling pathway

Endothelin signaling pathway

FGF signaling pathway

Glycolysis

Gonadotropin-releasing hormone receptor pathway

Hedgehog signaling pathway

Heterotrimeric G-protein signaling pathway-Gq alpha and Go alpha-mediated pathway

Huntington disease

Insulin/IGF pathway-mitogen-activated protein kinase kinase/MAP kinase cascade

Integrin signaling pathway

Ionotropic glutamate receptor pathway

Metabotropic glutamate receptor group I pathway

Metabotropic glutamate receptor group III pathway

Muscarinic acetylcholine receptor 1 and 3 signaling pathway

Oxytocin receptor-mediated signaling pathway

PDGF signaling pathway

Parkinson disease

Pyruvate metabolism 
Table 5 (continued)

\begin{tabular}{ll}
\hline Protein & Pathway (by PANTHER classification) \\
\hline $\begin{array}{l}\text { Ribosomal protein S6 kinase alpha-3 } \\
\text { (RPS6KA3) }\end{array}$ & Ras pathway \\
$\begin{array}{l}\text { Guanine nucleotide-binding protein subunit } \\
\text { alpha-11 (GNA11) }\end{array}$ & Thyrotropin-releasing hormone receptor signaling pathway \\
F-box/WD repeat-containing protein 1A & Toll pathway-drosophila \\
(BTRC) & \\
Ubiquitin/ISG15-conjugating enzyme E2 L6 & Ubiquitin proteasome pathway \\
(UBE2L6) & Vitamin D metabolism and pathway \\
Vitamin D-binding protein (GC) & Wnt signaling pathway \\
Myc proto-oncogene protein (MYC) & \\
F-box/WD repeat-containing protein 1A & \\
(BTRC) & \\
Guanine nucleotide-binding protein subunit & \\
alpha-11 (GNA11) & \\
Myc proto-oncogene protein (MYC) & p53 pathway feedback loops 2 \\
\hline
\end{tabular}

All spots were cut out from the 2D electrophoresis gels, washed, and destained for proteomic analysis. The subsequent reduction and alkylation processes were applied using dithiothreitol and iodoacetamide, respectively. After dehydration, gel pieces were incubated with trypsin in $50 \mathrm{mM}$ ammonium bicarbonate for overnight digestion at $37{ }^{\circ} \mathrm{C}$ (Promega, Trypsin Gold, Mass Spectrometry Grade, Technical Bulletin). After digestion, the peptides were extracted three times with $50 \mu \mathrm{l}$ of a solution containing ACN/H2O/TFA (500:450:50) with sonification for $15 \mathrm{~min}$ at room temperature in an ultrasonic water bath. The supernatant was collected and dried in the CentriVap (Labconco). Concentrated and purified peptides were obtained by employing $\mu \mathrm{C} 18$ Zip-TIP (PR 02358, Merck Technical Note) and finally transferred onto the MTP plate (Bruker).

MALDI-TOF was used as a soft ionization method because it only introduces a charge and does not cause fragmentation of the analyzed compound. The experiment was conducted in an ultrafleXtreme (Bruker) machine with a TOF/TOF detector to guarantee high accuracy and resolution of the measurements. All of the spectra were collected within the 900-4000 Da range in the active reflection mode, and this mass range was used to acquire the tandem mass spectrometry (MS/MS) spectra. HCCA (alpha-cyano-4-hydroxycinnamic acid, portioned; Bruker) was used as the matrix in the dried droplet method $(0.5 \mu$ l sample $+0.5 \mu$ l matrix $)$ following the standard manufacturer's protocol for peptide analysis. An MTP AnchorChip 384 (Bruker) with hydrophilic spots was used as the holder for sample preparation. Each sample was spotted onto three different active spots, and the profiled spectra were calibrated using the peptide mixture Peptide Calibration Standard I (Bruker). The flexControl program 3.3 (version 108) was used for mass spectra collection, flexAnalysis 3.3 (version 80) was used for analysis, and finally, the SwissProt database was searched using the software BioTools 3.2 (version 4.48). All spectra were systematically processed as follows: smoothing performed by the SavitskyGolay method, baseline subtraction was performed by the Top Hat baseline algorithm, peak geometry was characterized by the Stanford Network Analysis Platform (SNAP) algorithm,
Table 6 Proteins linked to Hsp90 according to Picard Laboratory

\begin{tabular}{llll}
\hline Protein & Wet AMD & Dry AMD & Control \\
\hline Annexin A3 & + & - & - \\
Myosin-13 & + & - & - \\
Insulin-like growth factor-binding protein 3 & + & - & - \\
Hsp90 co-chaperone Cdc37-like 1 & - & + & - \\
SRC kinase signaling inhibitor & + & + & - \\
Rho GTPase-activating protein 24 & + & + & - \\
Prolactin-inducible protein & + & + & + \\
$\beta$-Actin & + & + & + \\
Apolipoprotein A & + & - & - \\
Histones H2B & + & - & \\
\hline
\end{tabular}


Table 7 Proteins identified as a potential biomarkers in different studies

\begin{tabular}{|c|c|c|c|}
\hline $\begin{array}{l}\text { Proteins previously } \\
\text { identified }\end{array}$ & Wet $A M D$ group & Dry AMD group & $\begin{array}{l}\text { Article } \\
\text { no. }\end{array}$ \\
\hline Serum albumin & + & + & $1,5,6$ \\
\hline Apolipoprotein E & Apolipoprotein L6 & - & 1 \\
\hline $\begin{array}{l}\text { Actin beta (1) } \\
\text { Actin, aortic smooth } \\
\text { muscle ( } 2)\end{array}$ & Actin cytoplasmic 1 & Actin cytoplasmic 1 & 1,2 \\
\hline Annexin II & Annexin A3 & - & 1 \\
\hline $\begin{array}{l}\text { Histone H2B C; H2Ao; } \\
\text { H2A2 }\end{array}$ & Histone H1.4; H2A type 2-B & - & 1 \\
\hline Cystatin C & Cystatin S; SN & Cystatin S & 2 \\
\hline $\begin{array}{l}\text { Leucine-rich } \\
\text { repeat-containing pro- } \\
\text { tein } 15\end{array}$ & - & $\begin{array}{l}\text { Leucine-rich repeat-containing } \\
\text { protein } 16 \mathrm{~A}\end{array}$ & 2 \\
\hline Pyruvate kinase & - & + & 3 \\
\hline HLA-drb1-5 & $\begin{array}{l}\text { HLA Class I histocompatibility } \\
\text { antigen } \mathrm{Cw}-15 \text { alpha chain }\end{array}$ & & 4 \\
\hline $\begin{array}{l}\text { Fibroblast growth factor } \\
\quad 6\end{array}$ & Fibroblast growth factor 8 & Fibroblast growth factor 9 & 4 \\
\hline $\begin{array}{l}\text { Peroxiredoxin } 3 \text { isoform } \\
\beta\end{array}$ & Peroxiredoxin 6 & Peroxiredoxin 1 & 4 \\
\hline $\begin{array}{l}\text { NADH dehydrogenase } \\
1 \alpha\end{array}$ & $\begin{array}{l}\text { NADH dehydrogenase } 1 \alpha(4 \times \\
\text { subcomplex/subunit) }\end{array}$ & $\begin{array}{l}\text { NADH dehydrogenase } 1 \alpha(2 \times \\
\text { subcomplex/subunit) }\end{array}$ & 4 \\
\hline Apolipoprotein a-i; a-iv & Apolipoprotein L6 & - & 4,6 \\
\hline $\begin{array}{l}\text { Ubiqutin-conjugating } \\
\text { enzyme e2 }\end{array}$ & - & $\begin{array}{l}\text { Ubiquitin/ISG15-conjugating } \\
\text { enzyme E2 L6 }\end{array}$ & 4 \\
\hline $\begin{array}{l}\text { Vitamin D-binding pro- } \\
\text { tein }\end{array}$ & - & + & 4 \\
\hline Lipocalin-1 & + & - & 5 \\
\hline Serotransferrin & + & - & 5,6 \\
\hline
\end{tabular}

1 Drusen proteome analysis: an approach to the etiology of age-related macular degeneration. Crabb et al. Proc Natl Acad Sci USA. 2002 Nov 12;99(23):14682-7, 2 Proteomic analysis of the aqueous humor in age-related macular degeneration (AMD) patients. Kim et al. J Proteome Res. 2012 Aug 3;11(8):4034-43, 3 Proteomics of the retinal pigment epithelium reveals altered protein expression at progressive stages of AMD. Nordgaard et al. Invest Ophthalmol Vis Sci. 2006 Mar;47(3):815-22, 4 Comparative proteomic analysis of plasma proteins in patients with age-related macular degeneration. Xu et al. Int J Ophthalmol. 2014 Apr 18;7(2):256-63, 5 Proteomic analysis of the aqueous humor in patients with wet age-related macular degeneration. Yao et al. Proteomics Clin Appl. 2013 Aug;7(7-8):550-60, 6 Proteomics of vitreous humor of patients with exudative age-related macular degeneration. Koss et al. PLoS One. 2014 May 14;9(5):e96895 and all peaks with a signal ratio above 4 were qualified for further analysis. The parameters for the Mascot database search as follows: errors in both MS and MS/MS mode at 0.3 D38, global modification of carbamidomethyl (C), possible modification and oxidation (M)14, partials at 1, and trypsin enzyme. Spectra with peptide matches above 5 peaks were considered statistically significant, and only five proteins were identified with a single peptide match. All of the peptide mass fingerprint spectra were analyzed again in MS/MS mode to confirm their exact amino acid sequence.

\section{Results}

The tear film proteome reveals specific proteins that can be linked with AMD pathology. We analyzed all of the proteins cut from the electrophoretic gels and created a tear film proteome map for each study group. A total of 342 proteins were identified: 138 for wet AMD, 125 for dry AMD, and 126 for control group patients (Fig. 1). In our study, eight proteins were recognized exclusively for AMD, i.e., in the both wet and dry disease forms: shootin-1, histatin-3, fidgetin-like protein 1, SRC kinase signaling inhibitor, Graves disease carrier protein, actin cytoplasmic 1, prolactin-inducible protein 1, and protein S100-A7A. All of the proteins identified are shown in the supplementary material Tables 1, 2, 3 with obtained score, mass, matching number, and UniProt accession number for control group, wet AMD, and dry AMD, respectively. Fig. 1 shows the Venn diagram of all proteins identified and reveals how they overlap in different study groups according to Venny 2.0.1 application (Oliveros). The obtained data was analyzed using the PANTHER classification system (http://pantherdb. 
org) to obtain a functional classification with a special focus on metabolic pathways. Table 4 shows proteins for wet AMD and Table 5 for dry AMD. The wet AMD proteomic results reveal that there was an upregulation of proteins that regulate inflammation and neovascularization. Additionally, with respect to the growing understanding of importance of clearance mechanisms in AMD etiology, we added Hsp90related proteins found in tear film, based on the Picard laboratory database which is available online (https://www.picard. ch/downloads/Hsp90interactors.pdf) (Table 6). We observed that 17 of the analyzed proteins had been previously recognized as potential AMD biomarkers, i.e., they had been detected in aqueous humor, vitreous, blood plasma, central and peripheral retina, or drusen (Table 7).

\section{Discussion}

A total of 110 specific proteins were identified in the wet AMD samples. Interestingly, many of the discovered proteins are known to be involved in the regulation of phagocytosis, inflammation, proteolysis, or alternatively they play a key role in cytoskeletal function. Choroidal neovascularization is one of the hallmarks of wet AMD. New vessels sprout from the choroidal capillaries through Bruch's membrane into the subRPE space or into the retinal layers. The weakened structure of new vascular tissue causes leakage into the retinal layers. This promotes fibrogliosis, resulting in the formation of a disciform scar and severe visual loss if not treated with intravitreal anti-VEGF drugs. The upregulation of several proteins, e.g., VEGF, MMPs, IGF-1, has been linked to the pathology of neovascularization. We detected four tear film proteins involved in several angiogenesis-related pathways, i.e., the PDGF signaling pathway, the EGF signaling pathway, and the CCKR signaling pathway (also linked with cancer [53]) in wet AMD samples [54-56].

Inflammation is one of the best documented processes underlying the pathogenesis of AMD [57-59]. It is strongly associated with chronic oxidative stress and impairment of autophagy [3]. Short-lasting inflammation is beneficial in host defense in a process called para-inflammation, but chronic inflammation leads to the detrimental tissue alterations encountered in AMD and other age-related diseases [60, 61]. We observed upregulation of several inflammatory agents, e.g., RASA1, STAT3, and Myosin-13 in the tear film samples isolated from wet AMD patients. STAT3 has been demonstrated to enhance choroidal neovascularization, and this factor is known to be activated by elevated IL-10 levels [62]. STAT3 has been postulated to be a potential biomarker for diagnosis and treatment of AMD due to its regulatory role in the survival of RPE cells; it is also recognized as playing a role in the regulation of visual cycle and the inflammatory response [63].
RPE cell apoptosis is thought to be an important cell death mechanism in AMD [6]. It consists of two main pathways: the FasL-related extrinsic (death receptor) pathway and the intrinsic (mitochondrial) pathway [64]. Evidence has been obtained of enhanced apoptosis in post-mortem wet AMD samples [65]. Increased apoptosis was also observed in $\mathrm{Ccl} 2^{-/-}$ $/ \mathrm{Cx} 3 \mathrm{crl}^{-/-}$(DKO) mice as well as in ARPE-19 cells exposed to different AMD-like stresses [7]. Interestingly, we observed upregulation of five proteins involved in three apoptosisrelated pathways in tear samples isolated from our wet AMD patients. Taken together, it seems that the upregulated proteins involved in inflammation and choroidal neovascularization, as well as in apoptotic processes in RPE cells, can be detected in the tear film of wet AMD patients.

Dry AMD patients showed 97 specific proteins related to 44 pathway patterns. Dry AMD-related proteins were involved in more pathways than those identified in the wet AMD. The two most widely expressed pathways were the Wnt signaling pathway and the Huntington disease pathway. It is noteworthy that there was a major representation of proteins involved in oxidative stress, inflammation, and proteolysis, e.g., the autophagy-related PI3K pathway. Autophagy failure has been reported to be associated with AMD development $[6,18,66]$.

AMD is an example of a protein misfolding disease [3]. Heat-shock proteins (Hsps) attempt to refold misfolded proteins and restore protein homeostasis in RPE cells. Hsp90 is a member of the Hsp family that has many interacting targets within cells [67] (https://www.picard.ch/downloads/ Hsp90interactors.pdf). In our study, 10 proteins were associated with Hsp90; 3 of them was present only in wet AMD, 1 only in dry AMD, 4 in both forms of AMD, and 2 in both controls and wet AMD. This considerable number of Hsp90-related proteins emphasizes its active involvement in both types of AMD and thus may represent a possible drug target. Hsp90 has been linked to autophagy, a process known to decline in the late stage of AMD [3, 64, 65, 68, 69]. Its role in Alzheimer's disease [67] and cancer [70] has also been documented. In retina, a short-term inhibition of Hsp90 was demonstrated to promote the viability of cells and improve visual function [71], whereas its prolonged inhibition led to the degradation of its client proteins which may impair visual function [66]. Two Hsp90 client proteins - insulin-like growth factor-binding protein 3 and Annexin A3-have been closely related to the process of choroidal neovascularization [72].

We believe that this is the first comprehensive in-depth analysis of human tear film proteome in patients suffering from different types of AMD. It seems that certain specific proteins are expressed in the tear film, and those biomarkers can be linked with AMD pathology. Our study supports the current view of AMD as a disease where inflammation, oxidative stress, and impaired autophagy play crucial role, making those processes a target for potential future treatment. Due 
to limitations of this pilot study, further investigations on tear film in AMD should be conducted, involving larger patient groups and division into AMD subtypes.

Acknowledgements The authors warmly acknowledge Dr. Ewen MacDonald for the language revision.

We would also like to show our gratitude to Dr. Katarzyna MichalakZwierz for the technical assistance in the preparation of $2 \mathrm{D}$ electrophoresis.

Author contributions Design of the study: MW, SW); conduct of the study, data collection, analysis, and interpretation: MW, DW; manuscript preparation: MW, KK, SW; review of the manuscript: KK, JM, ŁA.

Funding The study was partially funded by National Science Centre, Poland (NCN), grant number 2017/25/N/NZ5/01875. The Kuopio University Hospital VTR grant (KK5503743) and the Academy of Finland (KK296840) provided financial support in the form of KK and MW funding. The sponsor had no role in the design or conduct of this research.

\section{Compliance with ethical standards}

Conflict of interest The authors declare that they have no conflict of interest.

Ethical approval All procedures performed in studies involving human participants were in accordance with the ethical standards of the institutional and/or national research committee and with the 1964 Helsinki declaration and its later amendments or comparable ethical standards.

Informed consent Informed consent was obtained from all individual participants included in the study.

Open Access This article is distributed under the terms of the Creative Commons Attribution 4.0 International License (http:// creativecommons.org/licenses/by/4.0/), which permits unrestricted use, distribution, and reproduction in any medium, provided you give appropriate credit to the original author(s) and the source, provide a link to the Creative Commons license, and indicate if changes were made.

\section{References}

1. Brandl C, Breinlich V, Stark KJ et al (2016) Features of age-related macular degeneration in the general adults and their dependency on age, sex, and smoking: results from the German KORA study. PLoS One 11:e0167181. https://doi.org/10.1371/journal.pone. 0167181

2. Gordois A, Cutler H, Pezzullo L et al (2012) An estimation of the worldwide economic and health burden of visual impairment. Glob Public Health 7:465-481. https://doi.org/10.1080/17441692.2011. 634815

3. Kaarniranta K, Sinha D, Blasiak J et al (2013) Autophagy and heterophagy dysregulation leads to retinal pigment epithelium dysfunction and development of age-related macular degeneration. Autophagy 9:973-984. https://doi.org/10.4161/auto.24546

4. Kaarniranta K, Salminen A, Haapasalo A et al (2011) Age-related macular degeneration (AMD): Alzheimer's disease in the eye? J Alzheimers Dis 24:615-631. https://doi.org/10.3233/JAD-2011101908
5. Lambert NG, Singh MK, ElShelmani H et al (2016) Risk factors and biomarkers of age-related macular degeneration. Prog Retin Eye Res 54:64-102. https://doi.org/10.1016/j.preteyeres.2016.04. 003

6. Kaarniranta K, Tokarz P, Koskela A et al (2017) Autophagy regulates death of retinal pigment epithelium cells in age-related macular degeneration. Cell Biol Toxicol 33:113-128. https://doi.org/10. 1007/s10565-016-9371-8

7. Wang Y, Shen D, Wang VM et al (2012) Enhanced apoptosis in retinal pigment epithelium under inflammatory stimuli and oxidative stress. Apoptosis Int J Program Cell Death 17:1144-1155. https://doi.org/10.1007/s10495-012-0750-1

8. Blasiak J, Glowacki S, Kauppinen A, Kaarniranta K (2013) Mitochondrial and nuclear DNA damage and repair in age-related macular degeneration. Int J Mol Sci 14:2996-3010. https://doi.org/ 10.3390/ijms 14022996

9. Plafker SM, O'Mealey GB, Szweda LI (2012) Mechanisms for countering oxidative stress and damage in retinal pigment epithelium. Int Rev Cell Mol Biol 298:135-177. https://doi.org/10.1016/ B978-0-12-394309-5.00004-3

10. Krohne TU, Stratmann NK, Kopitz J, Holz FG (2010) Effects of lipid peroxidation products on lipofuscinogenesis and autophagy in human retinal pigment epithelial cells. Exp Eye Res 90:465-471. https://doi.org/10.1016/j.exer.2009.12.011

11. Valapala $M$, Edwards $M$, Hose $S$ et al (2014) Increased lipocalin-2 in the retinal pigment epithelium of Cryba $1 \mathrm{cKO}$ mice is associated with a chronic inflammatory response. Aging Cell 13:1091-1094. https://doi.org/10.1111/acel.12274

12. Vives-Bauza C, Anand M, Shiraz AK et al (2008) The age lipid $\mathrm{A} 2 \mathrm{E}$ and mitochondrial dysfunction synergistically impair phagocytosis by retinal pigment epithelial cells. J Biol Chem 283:24770 24780. https://doi.org/10.1074/jbc.M800706200

13. Subrizi A, Toropainen E, Ramsay E et al (2015) Oxidative stress protection by exogenous delivery of rhHsp70 chaperone to the retinal pigment epithelium (RPE), a possible therapeutic strategy against RPE degeneration. Pharm Res 32:211-221. https://doi.org/ 10.1007/s11095-014-1456-6

14. Decanini A, Nordgaard CL, Feng X et al (2007) Changes in select redox proteins of the retinal pigment epithelium in age-related macular degeneration. Am J Ophthalmol 143:607-615. https://doi.org/ 10.1016/j.ajo.2006.12.006

15. Schutt F, Ueberle B, Schnölzer M et al (2002) Proteome analysis of lipofuscin in human retinal pigment epithelial cells. FEBS Lett 528: 217-221

16. Ryhänen T, Hyttinen JMT, Kopitz J et al (2009) Crosstalk between Hsp70 molecular chaperone, lysosomes and proteasomes in autophagy-mediated proteolysis in human retinal pigment epithelial cells. J Cell Mol Med 13:3616-3631. https://doi.org/10.1111/j. 1582-4934.2008.00577.x

17. Ferguson TA, Green DR (2014) Autophagy and phagocytosis converge for better vision. Autophagy 10:165-167. https://doi.org/10. 4161/auto.26735

18. Viiri J, Amadio M, Marchesi N et al (2013) Autophagy activation clears ELAVL1/HuR-mediated accumulation of SQSTM1/p62 during proteasomal inhibition in human retinal pigment epithelial cells. PLoS One 8:e69563. https://doi.org/10.1371/journal.pone.0069563

19. Saraygord-Afshari N, Naderi-Manesh H, Naderi M (2015) Increasing proteome coverage for gel-based human tear proteome maps: towards a more comprehensive profiling. Biomed Chromatogr 29:1056-1067. https://doi.org/10.1002/bmc.3392

20. Boehm N, Funke S, Wiegand M et al (2013) Alterations in the tear proteome of dry eye patients-a matter of the clinical phenotype. Invest Ophthalmol Vis Sci 54:2385-2392. https://doi.org/10.1167/ iovs.11-8751

21. Li B, Sheng M, Li J et al (2014) Tear proteomic analysis of Sjögren syndrome patients with dry eye syndrome by two-dimensional- 
nano-liquid chromatography coupled with tandem mass spectrometry. Sci Rep 4:5772. https://doi.org/10.1038/srep05772

22. Zhou L, Wei R, Zhao P et al (2013) Proteomic analysis revealed the altered tear protein profile in a rabbit model of Sjögren's syndromeassociated dry eye. Proteomics 13:2469-2481. https://doi.org/10. 1002/pmic.201200230

23. Kim H-J, Kim P-K, Yoo H-S, Kim C-W (2012) Comparison of tear proteins between healthy and early diabetic retinopathy patients. Clin Biochem 45:60-67. https://doi.org/10.1016/j.clinbiochem. 2011.10.006

24. Li B, Sheng M, Xie L et al (2014) Tear proteomic analysis of patients with type 2 diabetes and dry eye syndrome by twodimensional nano-liquid chromatography coupled with tandem mass spectrometry. Invest Ophthalmol Vis Sci 55:177-186. https://doi.org/10.1167/iovs.13-12080

25. Hagan S, Martin E, Enríquez-de-Salamanca A (2016) Tear fluid biomarkers in ocular and systemic disease: potential use for predictive, preventive and personalised medicine. EPMA J 7:15. https:// doi.org/10.1186/s13167-016-0065-3

26. Nordgaard CL, Berg KM, Kapphahn RJ et al (2006) Proteomics of the retinal pigment epithelium reveals altered protein expression at progressive stages of age-related macular degeneration. Invest Ophthalmol Vis Sci 47:815-822. https://doi.org/10.1167/iovs.050976

27. Ethen CM, Reilly C, Feng X et al (2006) The proteome of central and peripheral retina with progression of age-related macular degeneration. Invest Ophthalmol Vis Sci 47:2280-2290. https://doi. org/10.1167/iovs.05-1395

28. Koss MJ, Hoffmann J, Nguyen N et al (2014) Proteomics of vitreous humor of patients with exudative age-related macular degeneration. PLoS One 9:e96895. https://doi.org/10.1371/journal.pone. 0096895

29. Nobl M, Reich M, Dacheva I et al (2016) Proteomics of vitreous in neovascular age-related macular degeneration. Exp Eye Res 146: 107-117. https://doi.org/10.1016/j.exer.2016.01.001

30. Yao J, Liu X, Yang Q et al (2013) Proteomic analysis of the aqueous humor in patients with wet age-related macular degeneration. Proteomics Clin Appl 7:550-560. https://doi.org/10.1002/prca. 201200012

31. Kim TW, Kang JW, Ahn J et al (2012) Proteomic analysis of the aqueous humor in age-related macular degeneration (AMD) patients. J Proteome Res 11:4034-4043. https://doi.org/10.1021/ pr300080s

32. Iannaccone A, Neeli I, Krishnamurthy P et al (2012) Autoimmune biomarkers in age-related macular degeneration: a possible role player in disease development and progression. In: Retinal degenerative diseases. Springer, Boston, pp 11-16

33. Iannaccone A, Giorgianni F, New DD et al (2015) Circulating autoantibodies in age-related macular degeneration recognize human macular tissue antigens implicated in autophagy, immunomodulation, and protection from oxidative stress and apoptosis. PLoS One 10:e145323. https://doi.org/10.1371/journal. pone. 0145323

34. Cabral T, Lima LH, Mello LGM et al (2018) Bevacizumab injection in patients with Neovascular age-related macular degeneration increases angiogenic biomarkers. Ophthalmol Retina 2:31-37. https://doi.org/10.1016/j.oret.2017.04.004

35. Sakamoto S, Takahashi H, Tan X et al (2018) Changes in multiple cytokine concentrations in the aqueous humour of neovascular agerelated macular degeneration after 2 months of ranibizumab therapy. Br J Ophthalmol 102(4):448-454. https://doi.org/10.1136/ bjophthalmol-2017-310284

36. Kang G-Y, Bang JY, Choi AJ et al (2014) Exosomal proteins in the aqueous humor as novel biomarkers in patients with neovascular age-related macular degeneration. J Proteome Res 13:581-595. https://doi.org/10.1021/pr400751k
37. Ng DS, Yip YW, Bakthavatsalam M et al (2017) Elevated angiopoietin 2 in aqueous of patients with neovascular age related macular degeneration correlates with disease severity at presentation. Sci Rep 7:45081. https://doi.org/10.1038/srep45081

38. Kim H-J, Ahn SJ, Woo SJ et al (2016) Proteomics-based identification and validation of novel plasma biomarkers phospholipid transfer protein and mannan-binding lectin serine protease- 1 in age-related macular degeneration. Sci Rep 6:32548. https://doi. org $/ 10.1038 /$ srep32548

39. Adamus G, Chew EY, Ferris FL, Klein ML (2014) Prevalence of anti-retinal autoantibodies in different stages of age-related macular degeneration. BMC Ophthalmol 14:154. https://doi.org/10.1186/ 1471-2415-14-154

40. Crabb JW, Miyagi M, Gu X et al (2002) Drusen proteome analysis: an approach to the etiology of age-related macular degeneration. Proc Natl Acad Sci U S A 99:14682-14687. https://doi.org/10. 1073/pnas.222551899

41. Kim H-J, Woo SJ, Suh EJ et al (2014) Identification of vinculin as a potential plasma marker for age-related macular degeneration. Invest Ophthalmol Vis Sci 55:7166-7176. https://doi.org/10.1167/ iovs.14-15168

42. Xu X-R, Zhong L, Huang B-L et al (2014) Comparative proteomic analysis of plasma proteins in patients with age-related macular degeneration. Int J Ophthalmol 7:256-263. https://doi.org/10. 3980/j.issn.2222-3959.2014.02.12

43. Yuan X, Gu X, Crabb JS et al (2010) Quantitative proteomics: comparison of the macular Bruch membrane/choroid complex from age-related macular degeneration and normal eyes. Mol Cell Proteomics 9:1031-1046. https://doi.org/10.1074/mcp.M900523MCP200

44. Ecker SM, Pfahler SM, Hines JC et al (2012) Sequential in-office vitreous aspirates demonstrate vitreous matrix metalloproteinase 9 levels correlate with the amount of subretinal fluid in eyes with wet age-related macular degeneration. Mol Vis 18:1658-1667

45. Sung HJ, Han JI, Lee JW et al (2012) TCCR/WSX-1 is a novel angiogenic factor in age-related macular degeneration. Mol Vis 18: 234-240

46. Li K, Chen Z, Duan F et al (2010) Quantification of tear proteins by SDS-PAGE with an internal standard protein: a new method with special reference to small volume tears. Graefes Arch Clin Exp Ophthalmol Graefes Arch Clin Exp Ophthalmol Albrecht Von Graefes Arch Klin Exp Ophthalmol 248:853-862. https://doi.org/ 10.1007/s00417-009-1275-3

47. Posa A, Bräuer L, Schicht M et al (2013) Schirmer strip vs. capillary tube method: non-invasive methods of obtaining proteins from tear fluid. Ann Anat Anat Anz Off Organ Anat Ges 195:137-142. https://doi.org/10.1016/j.aanat.2012.10.001

48. Green-Church KB, Nichols KK, Kleinholz NM et al (2008) Investigation of the human tear film proteome using multiple proteomic approaches. Mol Vis 14:456-470

49. González N, Iloro I, Durán JA et al (2012) Evaluation of inter-day and inter-individual variability of tear peptide/protein profiles by MALDI-TOF MS analyses. Mol Vis 18:1572-1582

50. Rentka A, Koroskenyi K, Harsfalvi J et al (2017) Evaluation of commonly used tear sampling methods and their relevance in subsequent biochemical analysis. Ann Clin Biochem 54:521-529. https://doi.org/10.1177/0004563217695843

51. Ablamowicz AF, Nichols JJ (2017) Concentrations of MUC16 and MUC5AC using three tear collection methods. Mol Vis 23:529537

52. Shevchenko A, Wilm M, Vorm O, Mann M (1996) Mass spectrometric sequencing of proteins silver-stained polyacrylamide gels. Anal Chem 68:850-858

53. Tripathi S, Flobak $\AA$, Chawla K et al (2015) The gastrin and cholecystokinin receptors mediated signaling network: a scaffold for 
data analysis and new hypotheses on regulatory mechanisms. BMC Syst Biol 9:40. https://doi.org/10.1186/s12918-015-0181-z

54. Yu H, Lee H, Herrmann A et al (2014) Revisiting STAT3 signalling in cancer: new and unexpected biological functions. Nat Rev Cancer 14:736-746. https://doi.org/10.1038/nrc3818

55. Yu H, Pardoll D, Jove R (2009) STATs in cancer inflammation and immunity: a leading role for STAT3. Nat Rev Cancer 9:798-809. https://doi.org/10.1038/nrc2734

56. Zhang L, Zhan X, Yan D, Wang Z (2016) Circulating MicroRNA-21 is involved in lymph node metastasis in cervical cancer by targeting RASA1. Int J Gynecol Cancer Off J Int Gynecol Cancer Soc 26:810-816. https://doi.org/10.1097/ IGC.0000000000000694

57. Nita M, Grzybowski A, Ascaso FJ, Huerva V (2014) Age-related macular degeneration in the aspect of chronic low-grade inflammation (pathophysiological parainflammation). Mediat Inflamm 2014: 930671. https://doi.org/10.1155/2014/930671

58. Ozaki E, Campbell M, Kiang A-S et al (2014) Inflammation in agerelated macular degeneration. Adv Exp Med Biol 801:229-235. https://doi.org/10.1007/978-1-4614-3209-8_30

59. Stanton CM, Wright AF (2014) Inflammatory biomarkers for AMD. Adv Exp Med Biol 801:251-257. https://doi.org/10.1007/ 978-1-4614-3209-8 32

60. Franceschi $\mathrm{C}$, Bonafè $\mathrm{M}$, Valensin $\mathrm{S}$ et al (2000) Inflamm-aging. An evolutionary perspective on immunosenescence. Ann N Y Acad Sci 908:244-254

61. Kauppinen A, Paterno JJ, Blasiak J et al (2016) Inflammation and its role in age-related macular degeneration. Cell Mol Life Sci 73: 1765-1786. https://doi.org/10.1007/s00018-016-2147-8

62. Nakamura R, Sene A, Santeford A et al (2015) IL10-driven STAT3 signalling in senescent macrophages promotes pathological eye angiogenesis. Nat Commun 6:7847. https://doi.org/10.1038/ ncomms 8847

63. Patel AK, Syeda S, Hackam AS (2013) Signal transducer and activator of transcription 3 (STAT3) signaling in retinal pigment epithelium cells. JAK-STAT 2:e25434. https://doi.org/10.4161/ jkst.25434

64. Szatmári-Tóth M, Kristóf E, Veréb Z et al (2016) Clearance of autophagy-associated dying retinal pigment epithelial cells - a possible source for inflammation in age-related macular degeneration. Cell Death Dis 7:e2367. https://doi.org/10.1038/cddis.2016.133

65. Wang B, Chen Z, Yu F et al (2016) Hsp90 regulates autophagy and plays a role in cancer therapy. Tumour Biol J Int Soc Oncodevelopmental Biol Med 37:1-6. https://doi.org/10.1007/ s13277-015-4142-3

66. Klionsky DJ, Abdelmohsen K, Abe A et al (2016) Guidelines for the use and interpretation of assays for monitoring autophagy (3rd edition). Autophagy 12:1-222. https://doi.org/10.1080/15548627. 2015.1100356

67. Salminen A, Ojala J, Kaarniranta K et al (2011) Hsp90 regulates tau pathology through co-chaperone complexes in Alzheimer's disease. Prog Neurobiol 93:99-110. https://doi.org/10.1016/j.pneurobio. 2010.10.006

68. Samarasinghe B, Wales CTK, Taylor FR, Jacobs AT (2014) Heat shock factor 1 confers resistance to Hsp90 inhibitors through p62/ SQSTM1 expression and promotion of autophagic flux. Biochem Pharmacol 87:445-455. https://doi.org/10.1016/j.bcp.2013.11.014

69. Xiong R, Zhou W, Siegel D et al (2015) A novel Hsp90 inhibitor activates compensatory heat shock protein responses and autophagy and alleviates mutant A53T $\alpha$-synuclein toxicity. Mol Pharmacol 88:1045-1054. https://doi.org/10.1124/mol.115.101451

70. Miyata Y, Nakamoto H, Neckers L (2013) The therapeutic target Hsp90 and cancer hallmarks. Curr Pharm Des 19:347-365

71. Aguilà M, Bevilacqua D, McCulley $\mathrm{C}$ et al (2014) Hsp90 inhibition protects against inherited retinal degeneration. Hum Mol Genet 23: 2164-2175. https://doi.org/10.1093/hmg/ddt613

72. Zamora DO, Riviere M, Choi D et al (2007) Proteomic profiling of human retinal and choroidal endothelial cells reveals molecular heterogeneity related to tissue of origin. Mol Vis 13:2058-2065 